\section{Robustness Margins for Indirect Field-Oriented Control of Induction Motors}

\author{
A. S. Bazanella and R. Reginatto
}

\begin{abstract}
The influence of the rotor time constant mismatch on the stability of induction motors under indirect field-oriented control is analyzed. The results of [14] are generalized. A Lyapunov function which provides a global stability test and allows us to compute robustness margins is given. Different mechanisms for the loss of stability are detected by means of bifurcation analysis. Robustness margins and design guidelines are derived from these results.
\end{abstract}

\section{INTRODUCTION}

Indirect field-oriented control (IFOC) is a well established and widely applied control technique when dealing with high performance induction motor drives [37], [41], [40]. Yet, it was not until very recently that this control scheme has been provided a firm theoretical foundation [15], [23], [14].

The comissioning of an IFOC requires the knowledge of a single motor parameter, namely the rotor time constant, which can vary widely in practice [36], [22]. The robustness of this control strategy against mismatches in this parameter has been analyzed in [14], where the robust global stability of the operating point has been established from a qualitative standpoint. A range of the parameter mismatch that guarantees the uniqueness of the equilibrium point for any loading condition has also been given.

In this paper we analyze the effects of parameter mismatches on the behavior of induction motors under IFOC in speed regulation tasks. In Section II the system modeling and the control equations are given. Then we generalize and deepen the results of [14] in two directions. First, in Section III, the robustness of the global stability property is given a quantitative measure by means of a Lyapunov analysis similar to that in [14]. Second, in Section IV, a bifurcation analysis is presented in terms of two parameters, namely the motor load and the rotor time constant mismatch. Conditions for the ocurrence of saddle-node bifurcations are given, providing bounds on the two parameters that avoid such bifurcations and guarantee uniqueness of the equilibrium. Finally, a discussion of the results presented is given in Section V, deriving some design guidelines. All symbolic manipulations and plots presented have been performed with MATLAB V.

\section{Problem Statement}

The dynamic model of a current-driven induction motor expressing the rotor flux and the stator currents in a reference frame rotating at synchronous speed is given by [37], [41], [40]

$$
\begin{aligned}
\dot{x}_{1} & =-c_{1} x_{1}-u_{1} x_{2}+c_{2} u_{3} \\
\dot{x}_{2} & =-c_{1} x_{2}+u_{1} x_{1}+c_{2} u_{2} \\
\dot{w} & =-c_{3} w+c_{4}\left[c_{5}\left(x_{2} u_{3}-x_{1} u_{2}\right)-T_{m}\right]
\end{aligned}
$$

Manuscript received July 27, 1998; revised January 20, 1999 and July 7, 1999. Recommended by Associate Editor, L.-S. Wang.

A. S. Bazanella is on leave from the Department of Electrical Engineering, Universidade Federal do Rio Grande do Sul, Av. Osvaldo Aranha 103 , 90035-190, Porto Alegre, RS, Brazil.

R. Reginatto is with the Department of Automation and Systems, Universidade Federal de Santa Catarina, 88040-900 Florianópolis, SC, Brazil.

Publisher Item Identifier S 0018-9286(00)06022-0. where $x_{1}$ and $x_{2}$ represent, respectively, the quadracture axis component and the direct axis component of the rotor flux, $w$ is the rotor speed, $u_{1}, u_{2}$, and $u_{3}$ stand for the inputs - the slipping frequency, the direct axis stator current component, and the quadrature axis stator current component, respectively; $T_{m}$ is the load torque, which is assumed constant, and the " $c$ " parameters are all positive. In particular, $c_{1}$ represents the inverse of the rotor time constant, which is a critical parameter for indirect field-oriented control. The model considered in [14] is expressed in a reference frame fixed in the rotor and is, upon a nonsingular ( $u_{1}$-dependent) change of coordinates, equivalent to (1)-(3) with $c_{3}=0$. See [37], [41], and [40] for further details regarding the derivation of the induction motor model.

In speed regulation applications the indirect field-oriented control strategy is usually applied along with a PI speed loop. This control strategy is described by the following equations [37], [14]:

$$
\begin{aligned}
& u_{1}=\hat{c}_{1} \frac{u_{3}}{u_{2}} \\
& u_{2}=u_{2}^{0} \\
& u_{3}=k_{p}\left(w_{\text {ref }}-w\right)+k_{i} \int_{0}^{t}\left(w_{\text {ref }}(\zeta)-w(\zeta)\right) d \zeta
\end{aligned}
$$

where $\hat{c}_{1}$ is an estimate for the inverse rotor time constant $c_{1}, k_{p}$, and $k_{i}$ are the gains of the PI speed controller, $w_{\text {ref }}$ is the constant reference velocity and $u_{2}^{0}$ is some constant which defines the flux level.

If $\hat{c}_{1}=c_{1}$, that is, if we have a perfect estimate of the rotor time constant, we say that the control is tuned, otherwise it is said to be detuned. Accordingly, we define

$$
\kappa \triangleq \frac{\hat{c}_{1}}{c_{1}}
$$

as the degre of tuning. It is clear that $\kappa>0$ and the control is tuned if and only if $\kappa=1$.

The closed-loop system (1)-(3) with the control (4)-(6) is a fourthorder system that can be described as follows:

$$
\begin{aligned}
\dot{x}_{1}= & -c_{1} x_{1}+c_{2} x_{4}-\frac{\kappa c_{1}}{u_{2}^{0}} x_{2} x_{4} \\
\dot{x}_{2}= & -c_{1} x_{2}+c_{2} u_{2}^{0}+\frac{\kappa c_{1}}{u_{2}^{0}} x_{1} x_{4} \\
\dot{x}_{3}= & -c_{3} x_{3}-c_{4}\left[c_{5}\left(x_{2} x_{4}-u_{2}^{0} x_{1}\right)\right. \\
& \left.-T_{m}-\frac{c_{3}}{c_{4}} w_{\text {ref }}\right] \\
\dot{x}_{4}= & \left(k_{i}-k_{p} c_{3}\right) x_{3} \\
& -k_{p} c_{4}\left[c_{5}\left(x_{2} x_{4}-u_{2}^{0} x_{1}\right)-T_{m}-\frac{c_{3}}{c_{4}} w_{\text {ref }}\right]
\end{aligned}
$$

where we have defined the new state variables $x_{3} \triangleq w_{\text {ref }}-w$ and $x_{4} \triangleq u_{3}$.

We shall analyze the stability properties of the closed-loop system (8)-(11) and its dependence on the loading conditions and the degree of tuning $\kappa$.

\section{LYAPUNOV ANALYSIS}

Let us define a change of coordinates $z \triangleq x-x^{e}$, where $x^{e}=$ $\left[x_{1}^{e} x_{2}^{e} x_{3}^{e} x_{4}^{e}\right]^{T}$ represents a generic equilibrium point. The system can be described in these coordinates as

$$
\dot{z}_{1}=-c_{1} z_{1}+\left(c_{2}-\frac{x_{2}^{e} \kappa c_{1}}{u_{2}^{0}}\right) z_{4}-z_{2}\left(z_{4}+x_{4}^{e}\right) \frac{\kappa c_{1}}{u_{2}^{0}}
$$




$$
\begin{gathered}
\dot{z}_{2}=-c_{1} z_{2}+\frac{\kappa c_{1}}{u_{2}^{0}}\left[x_{1}^{e} z_{4}+z_{1}\left(z_{4}+x_{4}^{e}\right)\right] \\
\dot{z}_{3}=-c_{3} z_{3}-c_{4} c_{5}\left[z_{2}\left(z_{4}+x_{4}^{e}\right)-z_{1} u_{2}^{0}+x_{2}^{e} z_{4}\right] \\
\dot{z}_{4}=\left(k_{i}-k_{p} c_{3}\right) z_{3}-k_{p} c_{4} c_{5}\left[z_{2}\left(z_{4}+x_{4}^{e}\right)\right. \\
\left.\quad-z_{1} u_{2}^{0}+x_{2}^{e} z_{4}\right] .
\end{gathered}
$$

Define also, for convenience of notation

$$
\begin{aligned}
& \alpha \triangleq \frac{\kappa c_{1}}{u_{2}^{0} c_{4} c_{5}}>0 \\
& k_{2} \triangleq \frac{\alpha^{2} k_{i}}{c_{2}}>0 \\
& k_{3} \triangleq \alpha^{2} c_{3} \frac{k_{p}}{k_{i}}>0 .
\end{aligned}
$$

We will study the stability of the origin $z=0$ by means of the quadratic Lyapunov function candidate

$$
V(z)=\frac{1}{2} z^{T}\left(P_{1}+m P_{2}\right) z
$$

where

$$
\begin{aligned}
P_{1} & =\left[\begin{array}{cccc}
k_{p}^{2}+\frac{k_{2}}{\alpha} & 0 & -k_{2} & -k_{p} \alpha \\
0 & 0 & 0 & 0 \\
-k_{2} & 0 & k_{p}^{2} k_{3}+\alpha k_{2} & -k_{p} k_{3} \\
-k_{p} \alpha & 0 & -k_{p} k_{3} & k_{3}+\alpha^{2}
\end{array}\right] \\
P_{2} & =\left[\begin{array}{llll}
1 & 0 & 0 & 0 \\
0 & 1 & 0 & 0 \\
0 & 0 & 0 & 0 \\
0 & 0 & 0 & 0
\end{array}\right]
\end{aligned}
$$

and $m$ is a positive scalar to be assigned. The matrix $P_{1}+m P_{2}$ is symmetric positive definite for any $m>0$ and any possible operating condition.

The time derivative of this Lyapunov candidate can be calculated as

$$
\begin{aligned}
\dot{V}(z)= & -\alpha_{1} z_{1}^{2}-\alpha_{3} z_{3}^{2}-\alpha_{4} z_{4}^{2}-2 \beta_{13} z_{1} z_{3}+2 \beta_{14} z_{1} z_{4} \\
& -m c_{1} z_{1}^{2}-m c_{1} z_{2}^{2}+m \frac{x_{1}^{e} c_{1} \kappa}{u_{2}^{0}} z_{2} z_{4} \\
& +m\left(c_{2}-\frac{x_{2}^{e} c_{1} \kappa}{u_{2}^{0}}\right) z_{1} z_{4}
\end{aligned}
$$

where

$$
\begin{aligned}
\alpha_{1} & \triangleq \frac{c_{1}}{c_{2}}(\kappa+1)\left(k_{p}^{2} c_{2}+k_{i} \alpha\right)>0 \\
\alpha_{3} & \triangleq \frac{c_{3}}{c_{2}} \alpha^{2}\left(k_{p}^{2} c_{2}+k_{i} \alpha\right)>0 \\
\alpha_{4} & \triangleq c_{2} k_{p} \alpha>0 \\
\beta_{13} & \triangleq-\frac{\alpha}{2}\left[\frac{c_{1}}{c_{2}}(\kappa+1) k_{i} \alpha+\frac{c_{3}}{c_{2}}\left(k_{p}^{2} c_{2}+k_{i} \alpha\right)-k_{p} k_{i}\right] \\
\beta_{14} & \triangleq \frac{1}{2}\left[k_{i} \alpha+k_{p}^{2} c_{2}+k_{p} \alpha c_{1}(\kappa+1)\right]>0
\end{aligned}
$$

The Lyapunov derivative (19) can be put in a quadratic form as shown in (20), at the bottom of the page.

Define also the quadratic polynomial

$$
\begin{aligned}
p(m)= & p_{2} m^{2}+p_{1} m+p_{0} \\
p_{2}= & -c_{1} \alpha_{3}\left[\left(\frac{x_{1}^{e} c_{1} \kappa}{2 u_{2}^{0}}\right)^{2}+\left(\frac{c_{2} u_{2}^{0}-x_{2}^{e} c_{1} \kappa}{2 u_{2}^{0}}\right)^{2}\right] \\
p_{1}= & -\alpha_{1} \alpha_{3}\left(\frac{x_{1}^{e} c_{1} \kappa}{2 u_{2}^{0}}\right)^{2}-2 c_{1} \alpha_{3} \beta_{14}\left(\frac{c_{2} u_{2}^{0}-x_{2}^{e} c_{1} \kappa}{2 u_{2}^{0}}\right) \\
& +\beta_{13}^{2}\left(\frac{x_{1}^{e} c_{1} \kappa}{2 u_{2}^{0}}\right)^{2}+c_{1}^{2} \alpha_{3} \alpha_{4} \\
p_{0}= & c_{1}\left(\alpha_{1} \alpha_{3} \alpha_{4}-\alpha_{3} \beta_{14}^{2}-\beta_{13}^{2} \alpha_{4}\right) .
\end{aligned}
$$

Then we have the following result, which provides a robust stability test and establishes the robust global stability in the tuned condition.

Theorem 1: Let $p(m)$ have distinct real roots $m_{1}, m_{2}$ ordered such that $m_{2}>m_{1}$. If

$$
m_{2}>\frac{\beta_{13}^{2}-\alpha_{1} \alpha_{3}}{c_{1} \alpha_{3}} \triangleq m_{0}
$$

then the origin of the system (12)-(15) is globally asymptotically stable.

Proof: It is clear from (20) that if there exists an $m$ such that all the leading minors of the symmetric matrix $Q$ are positive then the origin will be globally asymptotically stable. The leading minors of first and second order are always positive, whereas the third and fourth order minors are given respectively by

$$
\begin{aligned}
& \Delta_{3}(m)=m c_{1}\left[\left(\alpha_{1}+m c_{1}\right) \alpha_{3}-\beta_{13}^{2}\right] \\
& \Delta_{4}(m)=m p(m) .
\end{aligned}
$$

If the roots of $p(m)$ are real and distinct, then $p(m)>0 \forall m \in$ ( $\left.m_{1}, m_{2}\right)$, since $p_{2}$ is negative; then $\Delta_{4}(m)$ is positive in this interval. On the other hand, $\Delta_{3}(m)>0, \forall m>m_{0}$, so that all the leading minors will be positive for $m \in\left(\max \left\{m_{0}, m_{1}\right\}, m_{2}\right)$. Condition (25) guarantees that this interval is not empty.

The robust global stability of the IFOC close to the tuned condition for general systems can be established as a direct consequence of Theorem 1.

Corollary 1: The equilibrium of the system (12)-(15) is globally asymptotically stable provided that $\kappa=1$ or sufficiently close to this value.

Proof: As the tuned condition $(\kappa=1)$ is approached, $p_{2}$ tends to zero by the left and $p_{1}$ tends to a finite positive value, since $x_{1}^{e}$ and $x_{2}^{e}$ approach 0 and $\left(c_{2} / c_{1}\right) u_{2}^{0}$, respectively. Hence one of the roots of $p(m)$ tends to $+\infty$ and, as $m_{0}$ remains finite, there exists an interval of values of $\kappa$ containing the point $\kappa=1$ such that condition (25) is satisfied.

$$
\left.\dot{V}(z)=-z^{T} Q z\right)=\left[\begin{array}{cccc}
\alpha_{1}+c_{1} m & 0 & \beta_{13} & -\beta_{14}-\frac{c_{2} u_{2}^{0}-x_{2}^{e} c_{1} \kappa}{2 u_{2}^{0}} m \\
0 & c_{1} m & 0 & -\frac{x_{1}^{e} c_{1} \kappa}{2 u_{2}^{0}} m \\
\beta_{13} & 0 & \alpha_{3} & 0 \\
-\beta_{14}-\frac{c_{2} u_{2}^{0}-x_{2}^{e} c_{1} \kappa}{2 u_{2}^{0}} m & -\frac{x_{1}^{e} c_{1} \kappa}{2 u_{2}^{0}} m & 0 & \alpha_{4}
\end{array}\right]
$$


Given the complexity of the expressions involved, it is impossible at this point to make general statements about the conditions of Theorem 1, unless in the important case of the corollary above. However, this theorem provides a simple robust stability test for any particular system, allowing us to establish robustness margins by the verification of condition (25) for different values of $\kappa$.

\section{BIFURCATION ANALYSIS}

\section{A. Parameterization of the Equilibria}

We start our analysis by studying the equilibria of the system (8)-(11) and their dependence on the degree of tuning $\kappa$. The equilibria of this system are the solutions $x_{1}^{e}, x_{2}^{e}, x_{3}^{e}, x_{4}^{e}$ of the system of equations

$$
\begin{aligned}
& -c_{1} x_{1}^{e}+c_{2} x_{4}^{e}-\frac{\kappa c_{1}}{u_{2}^{0}} x_{2}^{e} x_{4}^{e}=0 \\
& -c_{1} x_{2}^{e}+c_{2} u_{2}^{0}+\frac{\kappa c_{1}}{u_{2}^{0}} x_{1}^{e} x_{4}^{e}=0 \\
& -c_{3} x_{3}^{e}-c_{4}\left[c_{5}\left(x_{2}^{e} x_{4}^{e}-u_{2}^{0} x_{1}^{e}\right)-T_{m}-\frac{c_{3}}{c_{4}} w_{\text {ref }}\right]=0 \\
& \left(k_{i}-k_{p} c_{3}\right) x_{3}^{e}-k_{p} c_{4}\left[c_{5}\left(x_{2}^{e} x_{4}^{e}-u_{2}^{0} x_{1}^{e}\right)-T_{m}-\frac{c_{3}}{c_{4}} w_{\text {ref }}\right]=0
\end{aligned}
$$

The equilibrium values of the fluxes can be obtained rewriting (28) and (29) as

$$
\left[\begin{array}{cc}
c_{1} & c_{1} \kappa r \\
-c_{1} \kappa r & c_{1}
\end{array}\right]\left[\begin{array}{l}
x_{1}^{e} \\
x_{2}^{e}
\end{array}\right]=\left[\begin{array}{c}
\frac{1}{c_{2} u_{2}^{0}} r \\
c_{2} u_{2}^{0}
\end{array}\right]
$$

where we have defined the dimensionless variable $r \triangleq\left(x_{4}^{e} / u_{2}^{0}\right)$. Solving (32) for $x_{1}^{e}, x_{2}^{e}$ yields

$$
\begin{aligned}
& x_{1}^{e}=\frac{c_{2}}{c_{1}} u_{2}^{0} \frac{1-\kappa}{1+\kappa^{2} r^{2}} r \\
& x_{2}^{e}=\frac{c_{2}}{c_{1}} u_{2}^{0} \frac{1+\kappa r^{2}}{1+\kappa^{2} r^{2}} .
\end{aligned}
$$

Now, from (30) and (31) it is clear that

$$
\begin{aligned}
x_{3}^{e} & =0 \\
c_{5}\left(x_{2}^{e} r-x_{1}^{e}\right) u_{2}^{0} & =T_{e} \triangleq T_{m}+\frac{c_{3}}{c_{4}} w_{\text {ref }} .
\end{aligned}
$$

Substituting (33) and (34) into (36) we get

$$
\frac{c_{2}}{c_{1}}\left(u_{2}^{0}\right)^{2} \frac{\kappa r^{3}+\kappa r}{1+\kappa^{2} r^{2}}=\frac{T_{e}}{c_{5}} .
$$

Let us define also $r^{*} \triangleq\left(T_{e} c_{1} / c_{5} c_{2}\left(u_{2}^{0}\right)^{2}\right)$, which is a dimensionless quantity that represents the system loading, since it is proportional to the electrical torque developed in steady-state $T_{e}$. Also note that $r^{*}$ equals the value of $r$ in the tuned condition. Then, from (37), it follows that $r$ must satisfy the following polynomial equation:

$$
\kappa r^{3}-r^{*} \kappa^{2} r^{2}+\kappa r-r^{*}=0 .
$$

Collecting (33)-(35) and the definition of $r$ we can write the equilibrium point as

$$
\left[\begin{array}{l}
x_{1}^{e} \\
x_{2}^{e} \\
x_{3}^{e} \\
x_{4}^{e}
\end{array}\right]=\left[\begin{array}{cc}
\frac{c_{2} u_{2}^{0}}{c_{1}} & \frac{1-\kappa}{1+\kappa^{2} r^{2}} r \\
\frac{c_{2} u_{2}^{0}}{c_{1}} & \frac{1+\kappa r^{2}}{1+\kappa^{2} r^{2}} \\
0 \\
u_{2}^{0} r
\end{array}\right] .
$$

The equilibrium is now parameterized in terms of a single dimensionless quantity $r$, which satisfies (38). This is a third order polynomial equation whose coefficients-which are dimensionless as well — depend only on the degree of tuning $\kappa$ and the motor load as denoted by $r^{*}$.

\section{B. Uniqueness of the Equilibrium}

The real solutions of (38) give the equilibrium values of $r$ for any given degree of detuning $-\kappa$ - and any given load $-r^{*}$. It is clear that (38) has at least one and at most three real solutions, depending on the particular values of $\kappa$ and $r^{*}$

Theorem 2: Assume that

$$
\kappa>3
$$

and consider the inequalities shown in (41) and (42), at the bottom of the page.

If (41) and (42) are both satisfied strictly, then the system (8)-(11) has three equilibrium points. If either (41) or (42) holds with equality, then the system has two equilibrium points. Otherwise, the equilibrium point of this system is unique.

Proof: Rearranging (38) we have $r^{*}$ as a function of $r$

$$
r^{*}=f(r)=\kappa r \frac{r^{2}+1}{\kappa^{2} r^{2}+1} .
$$

Equation (38) has a unique real solution whenever $f(r)$ is bijective. It is clear that $f(r)$ is continuous and injective, so that it will be bijective if it is strictly monotonic

$$
\begin{aligned}
\frac{d f(r)}{d r} & =\kappa \frac{\left(\kappa^{2} r^{2}+1\right)\left(3 r^{2}+1\right)-r\left(r^{2}+1\right) 2 r \kappa^{2}}{\left(\kappa^{2} r^{2}+1\right)^{2}} \\
& =\kappa \frac{\kappa^{2} r^{4}+\left(3-\kappa^{2}\right) r^{2}+1}{\left(\kappa^{2} r^{2}+1\right)^{2}} .
\end{aligned}
$$

The sign of the derivative depends only on the sign of the polynomial in the numerator, since the denominator is always positive, as well as $\kappa$. Thus the function $f(r)$ is bijective for all values of $r^{*}$ such that

$$
\kappa^{2} r^{4}+\left(3-\kappa^{2}\right) r^{2}+1>0 .
$$

The points at which the derivative changes sign are the roots of the polynomial equation

$$
\kappa^{2} r^{4}+\left(3-\kappa^{2}\right) r^{2}+1=0 .
$$

$$
\begin{aligned}
& \left|r^{*}\right| \geq \frac{[\sqrt{(\kappa-1)(\kappa+3)}+\sqrt{(\kappa+1)(\kappa-3)}]\left[3\left(\kappa^{2}-1\right)+\sqrt{(\kappa-1)(\kappa+3)(\kappa+1)(\kappa-3)}\right]}{2 \kappa^{2}\left(\kappa^{2}-1+\sqrt{(\kappa-1)(\kappa+3)(\kappa+1)(\kappa-3)}\right)} \\
& \left|r^{*}\right| \leq \frac{[\sqrt{(\kappa-1)(\kappa+3)}+\sqrt{(\kappa+1)(\kappa-3)}]\left[3\left(1-\kappa^{2}\right)+\sqrt{(\kappa-1)(\kappa+3)(\kappa+1)(\kappa-3)}\right]}{2 \kappa^{2}\left(1-\kappa^{2}+\sqrt{(\kappa-1)(\kappa+3)(\kappa+1)(\kappa-3)}\right)}
\end{aligned}
$$




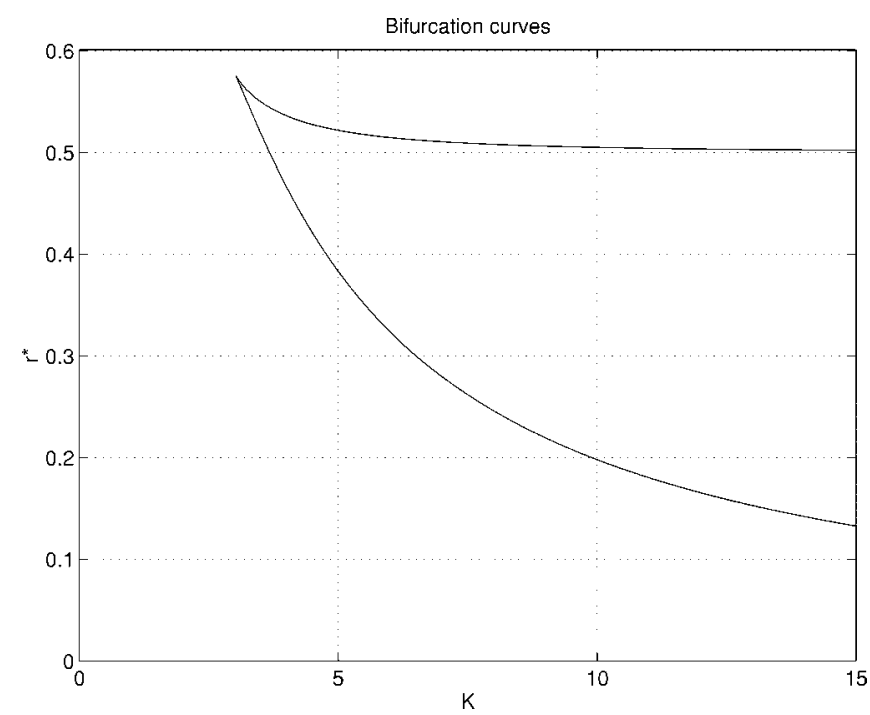

Fig. 1. Locus of the points in the parameter space where the number of equilibria changes.

The four solutions of this equation can be written as a function of $\kappa$

$$
r=\frac{1}{2 \kappa}[ \pm \sqrt{(\kappa-1)(\kappa+3)} \pm \sqrt{(\kappa+1)(\kappa-3)}]
$$

which yields real solutions only for $\kappa>3$, thus establishing the necessity of (40). In this case, two positive and two negative solutions exist. Since $f(\cdot)$ is odd, the analysis can be restricted to $r \in[0, \infty)$ and extended to negative $r$ by symmetry arguments. Let the positive solutions be $r_{1}$ and $r_{2}, r_{2}>r_{1}$; then the right-hand side of (41) and (42) are obtained by evaluating $f\left(r_{2}\right)$ and $f\left(r_{1}\right)$, respectively. By the sign of the derivative, it follows that $r_{1}$ is a local maximum and $r_{2}$ is a local minimum, and hence $f(r)$ will not be bijective for $r^{*}=$ $f(r) \in\left[f\left(r_{2}\right), f\left(r_{1}\right)\right]$. This is precisely the condition stated in (41) and (42). Indeed, the set $\left[\bar{r}_{1}, \bar{r}_{2}\right] \triangleq\left\{r: r^{*}=f(r) \in\left[f\left(r_{2}\right), f\left(r_{1}\right)\right]\right\}$ is well defined and such that $0<\bar{r}_{1}<r_{1}<r_{2}<\bar{r}_{2}$, since $f(\cdot)$ is continuous, unbounded, $f(r)>0, \forall r>0$, and $f(0)=0$. Observing that $f(\cdot)$ is strictly increasing in $A \triangleq\left(\bar{r}_{1}, r_{1}\right) \cup\left(r_{2}, \bar{r}_{2}\right)$ and strictly decreasing in $B \triangleq\left(r_{1}, r_{2}\right)$, it follows that (38) has three distinct solutions whenever $r^{*} \in\left(f\left(r_{2}\right), f\left(r_{1}\right)\right)$, thus establishing the first part of the theorem. Now, suppose (42) is satisfied with equality. Then, $r^{*}=f\left(r_{1}\right)$ and, since $f(\cdot)$ is strictly increasing in $\left(r_{2}, \infty\right)$, $f\left(\bar{r}_{2}\right)=f\left(r_{1}\right)$. No other solution exists since $r_{1}$ is a local maximum and $f(\cdot)$ also is strictly increasing in $\left[0, r_{1}\right)$. With a similar reasoning applied to the case in which (41) is satisfied with equality, the second part of the theorem follows. The theorem is proved by noting that $f$ is bijective outside the set $\left[\bar{r}_{1}, \bar{r}_{2}\right]$.

This result is ilustrated by Fig. 1, where the limits described by (41) and (42) are plotted. The point where the two curves intersect is $\kappa=3$, $r^{*}=(\sqrt{3} / 3)$. For large $\kappa$ the upper bound (42) tends to $r^{*}=0.5$ and the lower bound (41) tends to zero, as can be seen by analysis of (41) and (42). The system has three equilibria if the tuning and loading con- ditions lie in between the two curves and a unique equilibrium outside. At each one of the two curves two equilibria coincide.

\section{Local Stability Analysis}

The Jacobian matrix of the system (8)-(11) is given by (49), as shown at the bottom of the page, whose determinant is

$$
\operatorname{det}(J(\kappa, r))=c_{1} c_{2} c_{4} c_{5} u_{2}^{0} k_{i} \kappa \frac{\kappa^{2} r^{4}+\left(3-\kappa^{2}\right) r^{2}+1}{1+\kappa^{2} r^{2}} .
$$

We know from the stability analysis in the previous section that for the tuned condition the Jacobian has all its eigenvalues in the closed left half-plane. Accordingly, we can verify from (50) that $\operatorname{det}(J(1, r))>$ $0 \forall r$. As the parameters $\kappa$ and $r^{*}$ vary, loss of stability can be detected by the eigenvalues of the Jacobian, as either a pair of complex eigenvalues or a single real eigenvalue cross the imaginary axis toward the right half-plane. If a pair of complex eigenvalues crosses the imaginary axis, then a Hopf bifurcation takes place. Given the complexity of the expressions involved in an eigenvalue analysis, we cannot at this point provide generic conditions for the ocurrenc of Hopf bifurcations. We point out however, that it can happen for certain choices of PI gains, as shown by an example given in [14].

On the other hand, the crossing of a real eigenvalue through zero can be detected just by looking at the sign of $\operatorname{det}(J(\kappa, r))$ in (50). This determinant is the product of positive quantities and a fourth-order polynomial in $r$. Thus it is zero if and only if the polynomial is zero

$$
\kappa^{2} r^{4}+(3-\kappa) r^{2}+1=0 .
$$

Since this equation is the same which describes the points where the number of equilibria changes (47), the following fact has been established.

Fact 1: System (8)-(11) presents turning points (saddle-node bifurcations) at the solutions of (51).

Then the curves in Fig. 1 represent bifurcation surfaces in the parameter space, that is, they give the locus of all the turning points in this space [1], [2]. It is instructive to look at the branching diagram obtained varying $r^{*}$ for a given $\kappa$. Fig. 2 presents the branching diagram for $\kappa=4$. Two turning points can be seen, associated to the crossing of each one of the two curves in Fig. 1. This branch topology is known to cause the occurrence of jumps and hysteresis [2].

An additional local stability property follows from the above development.

Fact 2: Assume no Hopf bifurcation takes place for a given positive range of values of $r^{*}$ and $\kappa$. Then system (8)-(11) has at least one locally exponentially stable equilibrium point for all positive values of $\kappa$ and $r^{*}$ within this range. Moreover, equilibria such that (46) is satisfied will be locally exponentially stable, whereas other equilibria will be unstable.

By Theorem 1 it is clear that there exists a nonempty range of parameters around the tuned condition which satisfies the assumption of Fact 2. The occurrence of Hopf bifurcations depends on the setting of the PI speed controller. Conditions on the parameters $k_{p}$ and $k_{i}$ to avoid the Hopf bifurcation in the zero load torque case have been given in [14]. For small load torque the behavior is expected to be similar, so

$$
J(\kappa, r)=\left[\begin{array}{cccc}
-c_{1} & -c_{1} \kappa r & 0 & c_{2}(1-\kappa) /\left(1+\kappa^{2} r^{2}\right) \\
c_{1} \kappa r & -c_{1} & 0 & c_{2} \kappa(1-\kappa) /\left(1+\kappa^{2} r^{2}\right) r \\
c_{4} c_{5} u_{2}^{0} & -c_{4} c_{5} u_{2}^{0} r & -c_{3} & -c_{4} c_{5} \frac{c_{2}}{c_{1}} u_{2}^{0}\left(1+\kappa r^{2}\right) /\left(1+\kappa^{2} r^{2}\right) \\
k_{p} c_{4} c_{5} u_{2}^{0} & -k_{p} c_{4} c_{5} u_{2}^{0} r & k_{i}-k_{p} c_{3} & -k_{p} c_{4} c_{5} \frac{c_{2}}{c_{1}} u_{2}^{0}\left(1+\kappa r^{2}\right) /\left(1+\kappa^{2} r^{2}\right)
\end{array}\right]
$$




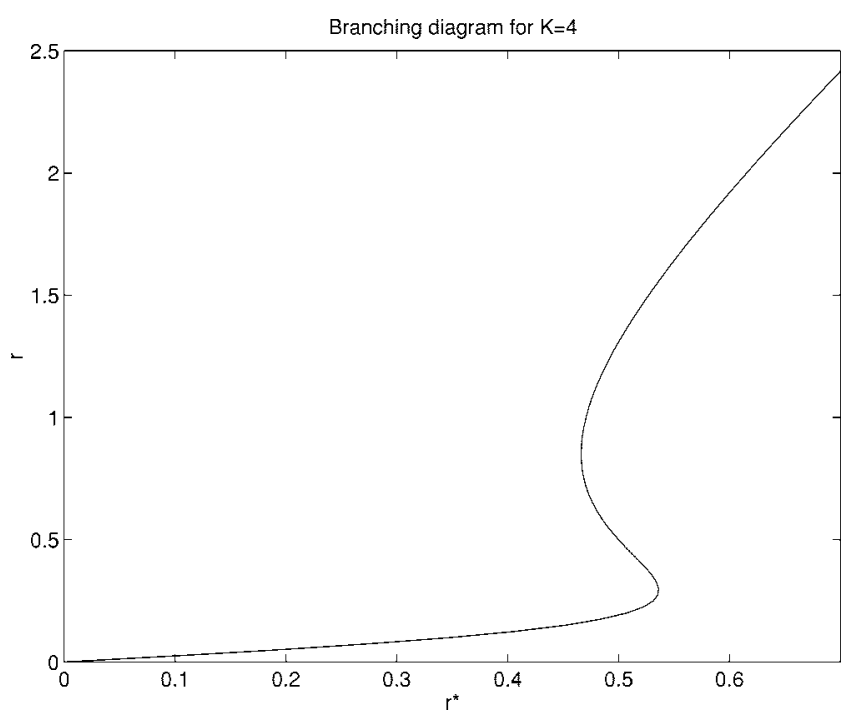

Fig. 2. Branching diagram for $\kappa=4$.

these conditions should be useful also for this case. However, it is still unclear how to predict and avoid the occurrence of Hopf bifurcations for higher loading conditions.

\section{DISCUSSION}

The conditions for saddle-node bifurcations in Section $\mathrm{V}$ are given in terms of the normalized load/flux rate $r^{*}$ and the normalized parameter mismatch $\kappa$. The range of parameters for which these bifurcations occur represents a low load/flux rate, which implies that operation under high flux yields smaller stability margins regarding saddle-node bifurcations. In any case, no saddle-node bifurcation occurs for $\kappa<$ 3 . Hence, in terms of robustness alone, it might be a good idea to "aim low" at the parameter $c_{1}$ when commissioning an IFOC, thus preventing a high value of $\kappa$. From this point of view it is also advisable to use low flux levels in order to avoid the saddle-node bifurcations.

When three equilibria are present, and under the assumptions of Fact 2 , two equilibria are locally asymptotically stable and the third one is unstable-Fact 3. The stable equilibrium which appears with low $r$ in the branch diagram (Fig. 2) is the operating point, and the other stable equilibrium presents a much larger current level. In actual operation, as the load varies passing through a saddle-node bifurcation the system will jump from the operating point to the other equilibrium.

Some simulations are provided to illustrate the system behavior for different mismatches in the rotor time constant. The model parameters for the case simulated are $c_{1}=13.67 \mathrm{~s}^{-1}, c_{2}=1.56 \mathrm{H} \cdot \mathrm{s}^{-1}, c_{3}=$ $0.59 \mathrm{~s}^{-1}, c_{4}=1,176 \mathrm{~kg}^{-11} \mathrm{~m}^{-2}, c_{5}=2.86$, taken from a $1-\mathrm{cv}$ squirrel-cage induction motor. The simulations presented in Figs. 3 and 4 show the system's behavior with $\kappa=1$ and $\kappa=4$ as the load torque is slowly increased from $T_{m}=0$ up to $T_{m}=4 \mathrm{~N} \cdot \mathrm{m}$. In the tuned condition the system behaves properly, but for $\kappa=4$ the loss of the equilibrium at which the system was operating causes a jump to a much higher current level. Although speed regulation is not lost in the simulation, in actual operation the motor would most likely stall due to overcurrent protection actuation. It is also worth noticing that even before the jump occurs the system may experience stability problems, since the existence of a nearby unstable equilibrium reduces the size of the region of attraction of the operating point.

Global stability of the IFOC has also been studied in this paper. A new Lyapunov function, which generalizes the one given in [14], has been given in Section III. By means of this Lyapunov function, a test for the robust global stability in different operating conditions has been
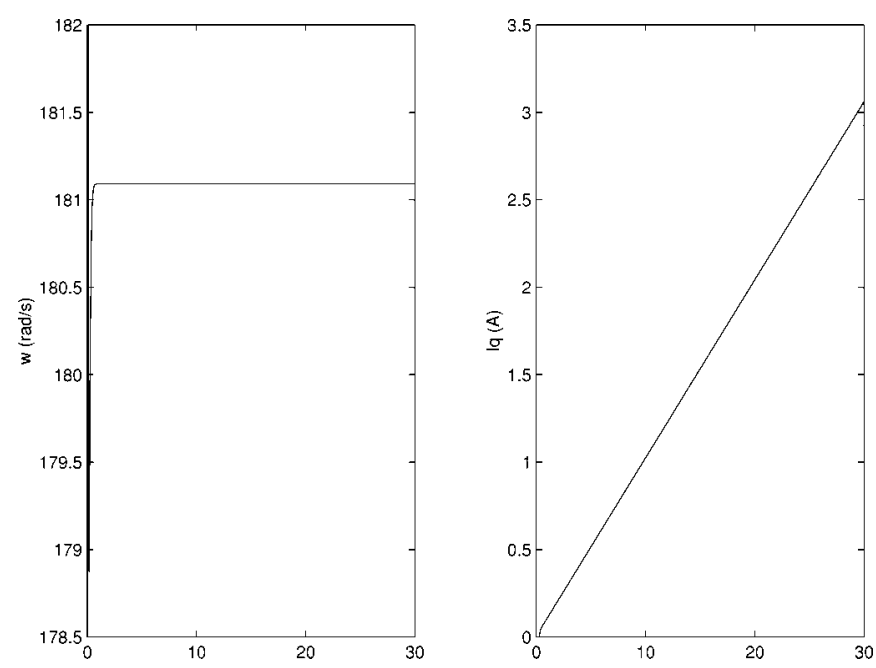

Fig. 3. Simulation for varying load torque in the tuned condition.
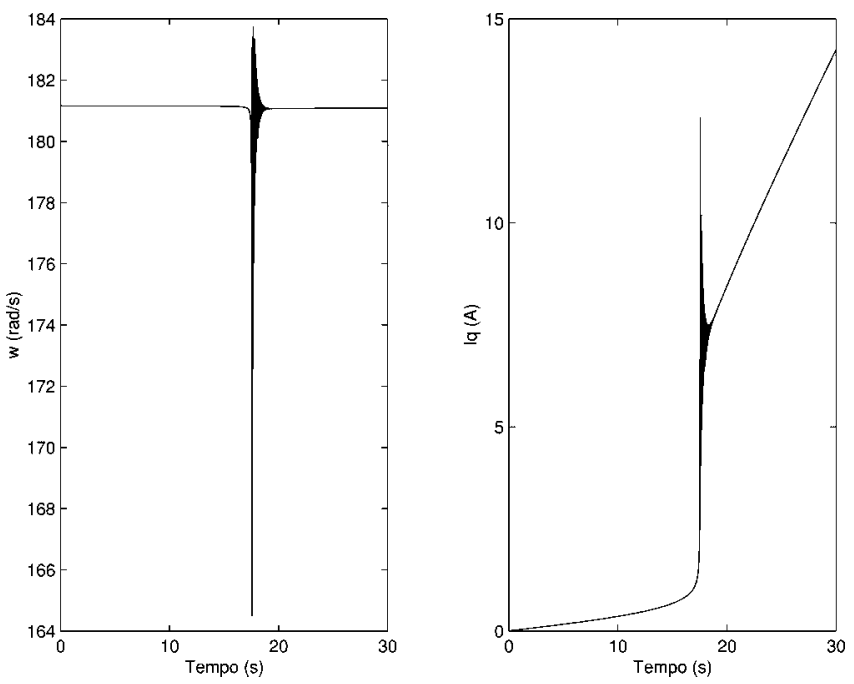

Fig. 4. Simulation for varying load torque with $\kappa=4$.

derived. The robustness property given in [14] has been given a quantitative measure, applicable for any given system.

These results reveal important structural stability/robustness properties of the IFOC. From a practical point of view, in most cases temperature variations inside the rotor can cause the rotor time constant to vary more than $50 \%$ but not more than $100 \%$ [36]. Hence, $\kappa<2$ in most practical cases, so that the robust global stability in the tuned condition along with the uniqueness of the equilibrium for $\kappa<3$ have strong practical implications. Yet, aiming low at $c_{1}$ and at the flux level may still be advisable in practical IFOC comissioning in order to keep the saddle-node bifurcation as far as possible. Closeness to a saddle-node bifurcation tends to deteriorate the system's performance and efficiency. Furthermore, much larger degrees of tuning $\kappa$ are likely to temporarily occur when adaptive techniques (like [31]) are employed, reaching the region where multiple equilibria are present. Thus, the results also have practical implications regarding the stability analysis and the design of adaptive IFOC drives.

It is important to stress that neither the existence of the robust global stability property in the tuned condition nor the bifurcation topology given in Section IV depend on the design of the PI controller. On the other hand, both the size of the robustness margin as given in Theorem 1 and the occurrence of Hopf bifurcations depend on the PI parameters $k_{p}$ 
and $k_{i}$. Conditions to be satisfied by these parameters in order to guarantee stability have been given in [14] for the zero load case only. The study of a number of different examples suggests that Hopf bifurcations are avoided by a correct classical PI tuning, but this is still an open issue.

\section{REFERENCES}

[1] J. Guckenheimer and P. Holmes, Nonlinear Oscillations, Dynamical Systems and Bifurcations of Vector Fields, 2nd ed. New York: Springer-Verlag, 1986

[2] R. Seydel, Practical Bifurcation and Stability Analysis: From Equilibrium to Chaos, 2nd ed. New York: Springer-Verlag, 1994.

[3] R. Chen and P. P. Varaiya, "Degenerate Hopf bifurcations in power systems," IEEE Trans. Circuits Syst., vol. 35, pp. 818-824, July 1988.

[4] H. O. Wang, E. H. Abed, and A. M. A. Hamdan, Voltage collapse dynamics and control in a sample power system,

[5] V. Ajjarapu and B. Lee, Bifurcations theory and its application to nonlinear dynamical phenomena in an electrical power system, .

[6] E. H. Abed, Control of bifurcations associated with voltage instability,

[7] A. I. Mees and L. O. Chua, "The Hopf bifurcation theorem and its applications to nonlinear oscillations in circuits and systems," IEEE Trans. Circuits Syst., vol. CAS-26, pp. 235-254, Apr. 1979.

[8] R. Aloliwi, E. G. Strangas, and H. K. Khalil, "Robust speed control of induction motors," in American Control Conf., Albuquerque, NM, June 1997, pp. 955-960.

[9] R. Noguchi, S. Kondo, and I. Takahashi, "Field-oriented control of an induction motor with robust on-line tuning of its parameters," IEEE Trans. Indust. Applicat., vol. 33, pp. 35-42, Jan./Feb. 1997.

[10] C. C. De Wit and S. I. Seleme, "Robust torque control design for induction motors: The minimum energy approach," Automatica, vol. 33, no. 1, pp. 63-79, 1997.

[11] C. Caminhas, R. Takahashi, P. Peres, and H. Tavares, "Observadores de Estados do Motor de Indução com Rejeição de Perturbações Paramétricas," in XII CBA-Congresso Brasileiro de Automática, Rio de Janeiro, 1996, pp. 1125-1130.

[12] P. Vedagarbha, D. M. Dawson, and R. Burg, "Velocity tracking/setpoint control of induction motors with improved efficiency," in IFAC 13th Triennial World Congr., San Francisco, CA, USA, 1996, pp. 37-42.

[13] R. J. Kerkman et al., "A new flux and stator resistence identifier for AC drive systems," IEEE Trans. Indus. Applicat., vol. 32, pp. 585-593, May/June 1996

[14] P. A. S. De Wit, R. Ortega, and I. Mareels, "Indirect field-oriented control of induction motors is robustly globally stable," Automatica, vol. 32, no. 10, pp. 1393-1402, 1996.

[15] R. Ortega, P. J. Nicklasson, and G. E. Péres, "On speed control of induction motors," Automatica, vol. 32, no. 3, pp. 455-460, 1996.

[16] S. H. Kim and S. K. Sul, "Maximum torque control of an induction machine in the field weakening region," IEEE Trans. Indus. Applicat., vol. 31, pp. 787-794, July/Aug. 1995.

[17] D. Borgard, G. Olsson, and R. D. Lorenz, "Accuracy issues for parameter estimation of field oriented induction machine drives," IEEE Trans Indus. Applicat., vol. 31, pp. 795-801, July/Aug. 1995.

[18] K. Ide et al., "Vector approximation method with parameter adaptation and torque control of CSI-Fed induction motor," IEEE Trans Indus. Applicat., vol. 31, pp. 830-840, July/Aug. 1995.

[19] R. W. De Doncker and D. W. Novotny, "The universal field oriented controller," IEEE Trans. Indus. Applicat., vol. 30, pp. 92-100, Jan./Feb. 1994.

[20] L. Huang, Y. Tadokoro, and K. Matsuse, "Deadbeat flux level control of direct-field-oriented high-horsepower induction servo motor using adaptive rotor flux observer," IEEE Trans. Indus. Applicat., vol. 30, pp. 954-962, July/Aug. 1994.

[21] H. Kubota and K. Matsuse, "Speed sensorless field-oriented control of induction motor with rotor resistence adaptation," IEEE Trans. Indus. Applicat., vol. 30, pp. 1219-1224, Sept./Oct. 1994

[22] R. Marino, S. Peresada, and P. Valigi, "Adaptive input-output linearizing control of induction motor," IEEE Trans. Automat. Contr., vol. 38, pp. 208-221, Feb. 1993.

[23] R. Ortega, C. Canudas, and S. I. Seleme, "Nonlinear control of induction motors: Torque tracking with unknown load disturbance," IEEE Trans. Automat. Contr., vol. 38, pp. 1675-1680, Nov. 1993.
[24] R. Ortega, C. Canudas, and S. I. Seleme, "Nonlinear control of induction motors: Torque tracking with unknown load disturbance," in American Control Conf., 1992, pp. 206-210.

[25] L. Zai, C. L. DeMarco, and T. A. Lipo, "An extended Kalman filter approach to rotor time constant measurement in PWM induction motor drives," IEEE Trans. Indus. Applicat., vol. 28, pp. 96-104, Jan./Feb. 1992.

[26] T. Ohtani, N. Takada, and K. Tanaka, "Vector control of induction motor without shaft encoder," IEEE Trans. Indus. Applicat., vol. 28, pp. 157-164, Jan./Feb. 1992.

[27] R. J. Kerkman, T. M. Rowman, and D. Leggate, "Indirect field-oriented control of an induction motor in the field-weakening region," IEEE Trans. Indus. Applicat., vol. 28, pp. 850-857, July/Aug. 1992.

[28] E. Bassi et al., "A field orienttion scheme for current-fed induction motor drives based on the torque angle closed-loop control," IEEE Trans. Indus. Applicat., vol. 28, pp. 1038-1044, Sept./Oct. 1992.

[29] Y. Kuroe, "Design of a new controller for induction motors based on exact linearization," in Proc. IECON'91-Industrial Electronics Conf., Oct./Nov. 1991, pp. 621-626.

[30] R. Krishnan and A. S. Bharadwaj, "A review of parameter sensitivity and adaptation in indirect vector controlled inductior motor drive systems," IEEE Trans. Indus. Applicat., vol. 6, pp. 695-703, Oct. 1991.

[31] T. M. Rowan, R. J. Kerkman, and D. Leggate, "A simple on-line adaption for indirect field orientation of an induction machine," IEEE Trans. Indus. Applicat., vol. 27, pp. 720-727, July/Aug. 1991.

[32] G. O. Garcia, R. M. Stephan, and E. H. Watanabe, "Controle de Velocidade de um Motor de Induccão através das Técnicas Campo Orientado Indireto e Escorregamento Controlado," in Anais do $8^{\circ}$ Congresso Brasileiro de Automática, UFPA-Belém, PA, 1990, pp. 955-960.

[33] A. de Luca and G. Ulivi, "Design of an exact nonlinear controller for induction motors," IEEE Trans. Automat. Contr., vol. 34, pp. 1304-1307, Dec. 1989.

[34] S. Ogasawara, H. Akagi, and A. Nabae, "The generalized theory of indirect vector control for AC machines," IEEE Trans. Indus. Applicat., vol. 24, pp. 470-478, May/June 1988.

[35] E. Y. Y. Ho and P. C. Sen, "Decoupling control of induction motor drives," IEEE Trans. Indus. Electron., vol. IE-35, pp. 253-262, May 1988.

[36] R. Krishnan and F. C. Doran, "Study of parameter sensitivity in highperformance inverter-fed induction motor drive systems," IEEE Trans. Indus. Applicat., vol. IA-23, pp. 623-635, July/Aug. 1987.

[37] D. W. Novotny and P. C. Sen, "Introduction to field orientation and high performance AC drives," in IEEE Indus. Applicat. Soc. Annual Meeting, Sept. 1986.

[38] L. J. Garcés, "Parameter adaption for the speed-controlled static AC drive with a squirrel-cage induction motor," IEEE Trans. Indus. Applicat., vol. IA-16, pp. 173-178, Mar./Apr. 1980

[39] F. Blaschke, "The principle of field orientation as applied to the new transvektor closed-loop control system for rotating-field machines," Siemens Rev., no. 5, pp. 217-220, 1972.

[40] B. K. Bose, Power Electronics and AC Drives. Englewood Cliffs, NJ: Prentice-Hall, 1987.

[41] W. Leonhard, Control of Electrical Drives. Berlin: Springer-Verlag, 1985. 